Ann. Biol. anim., Bioch., Biophys., r965, 5 (1), 6г - $7^{8}$.

\title{
ÉTUDE STATISTIQUE DES VARIATIONS DE L'EXCRÉTION D'AZOTE MÉTABOLIQUE FÉCAL ET D'AZOTE ENDOGÈNE URINAIRE EN FONCTION DU POIDS CORPOREL, DU POIDS DES INGESTA ET DU POIDS DES FÈCES CHEZ LE JEUNE RAT
}

\author{
I. - EN RÉGIME PROTÉIPRIVE
}

\author{
J. CAUSERET, Denise HUGOT et J. ARNOUX \\ Laboratoire d'Étude des Qualités biologiques des Aliments de l'Homme, \\ I.N.R.A., Centre national de Recherches zootechniques, Jouy-en-Josas (Seine-et-Oise) \\ et Service de Biométrie de l'I. N. R. A., \\ Centre national de Recherches agronomiques, Versailles (Seine-et-Oise)
}

SOMMAIRE;

L'influence du poids corporel, du poids de matière sèche ingérée et du poids de matière sèche fécale sur l'excrétion d'azote a été étudiée statistiquement sur ro groupes de jeunes rats mâles pesant initialement de 70 à $\mathrm{I} 30$ grammes, et soumis à un régime protéiprive. Ces groupes, qui comprenaient au total 4 I 7 sujets, ont été mis en expérience à des dates diverses, échelonnées sur une période de quatre années.

a) Dans chaque groupe, il existe une corrélation positive cntre l'excrétion d'azote métabolique fécal et chacune des trois variables indépendantes énumérées ci-dessus ; mais c'est toujours la corrélation entre l'azote métabolique fécal et le poids des fèces qui est la plus significative. D'autre part, quelle que soit la variable indépendante retenue, il existe une loi de régression commune à l'ensemble des groupes.

En outre, lorsqu'on a déjà considéré l'infuence de l'une quelconque des trois variables indépendantes, il y a intérêt à tenir compte aussi des deux autres, en estimant l'excrétion d'azote métabolique fécal sur la base d'une équation de régression triple.

Enfin, que l'excrétion soit estimée en fonction d'une seule ou cles trois variables indépendantes, on augmente la précision des calculs en substituant aux équations générales établies sur l'ensemble des animaux, des équations particulières à chaque groupe expérimental : ces équations ne différent les unes des autres que par la valeur du facteur constant.

b) Dans un certain nombre de groupes, il existe une corrélation positive entre l'excrétion d'azote endogène urinaire et le poids corporel ou le poids de matière sèche ingérée ; mais c'est la corrélation avec le poids corporel qui est la plus significative. Pour les deux variables indépendantes, il existe d'autre part une loi de régression commune à l'ensemble des groupes.

Mais, l'excrétion d'azote endogène urinaire est estimée avec plus de précision sur la base d'une équation de régression double, en tenant compte à la fois de l'influence du poids corporel et du poids de matière sèche ingérée. 
Enfin, il y a intérét à substituer aux équations générales établies sur l'ensemble des animaux, des équations particulières à chaque groupe expérimental, ces équations ne différant les unes des autres que par la valeur du facteur constant.

Toutefois, il n'a pas été possible d'obtenir une fonction permettant d'évaluer l'excrétion urinaire avec autant de précision que l'excrétion fécale. On peut se demander si les bases d'estimation les plus satisfaisantes ne seraient pas la quantité d'eau ingérée et le volume urinaire : cette éventualité est envisagée dans des essais en cours.

c) Il apparaît possible d'utiliser les données relatives au calcul de l'excrétion d'azote métabolique fécal pour la détermination du coefficient d'utilisation digestive réel et de la valeur biologique des protéines. En revanche, l'application des données relatives au calcul de l'excrétion d'azote endogène urinaire peut conduire à des erreurs appréciables.

\section{INTRODUCTION}

L'évaluation de l'utilisation digestive " nette " des protéines et de leur valeur biologique par les méthodes biologiques implique l'estimation de l'excrétion d'azote métabolique fécal et d'azote endogène urinaire. Cette détermination n'étant pas réalisable dans des conditions d'alimentation normales, on se contente généralement de travailler sur des sujets soumis, soit à un régime protéiprive, soit à un régime à 3-4 p. Ioo de protéines d'œuf (MitcheLI, I948).

Mais les pertes d'azote métabolique fécal et d'azote endogène urinaire peuvent varier beaucoup d'une expérience à une autre, et même d'un sujet à un autre dans une même expérience. Aussi divers auteurs se sont-ils intéressés aux facteurs dont ces pertes peuvent dépendre : âge et poids corporel, composition du régime alimentaire, quantité de matière sèche ingérée, poids sec des fèces, etc.

I $^{\circ}$ Chez le Rat, Mitcher. (I923-I924) a soutenu, dès ses premières recherches sur l'évaluation de la valeur biologique des protéines, que l'excrétion d'azote métabolique fécal est approximativement proportionnelle à la quantité de matière sèche ingérée : en I926, il a établi, sur des animaux soumis à un régime privé de protéines, la valeur moyenne de $\mathrm{I}, 9 \mathrm{mg}$ d'azote par gramme sec ingéré. Contesté par BoAs FIXSEN et JACKSON (I932), le point de vue de MITCHELI a été en partie confirmé par SCHNEIDER (I934), puis par CHICK et al. (I935), qui distinguent deux fractions dans 1'azote métabolique fécal :

- l'une, proportionnelle à la quantité de matière sèche ingérée,

- l'autre, indépendante des ingesta, mais vraisemblablement liée au poids corporel.

Mais ces auteurs n'en admettent pas moins la possibilité de rapporter de manière approximative l'excrétion d'azote métabolique fécal au gramme de matière sèche ingérée, sous réserve que la consommation de nourriture atteigne au moins quelques grammes par jour.

A plusieurs reprises, MrTchel, a tenté par la suite de faire la synthèse des résultats obtenus dans son laboratoire et d'en tirer des conclusions applicables à l'évaluation de l'utilisation digestive réelle des protéines. Dans une mise au point classique (I948), il cite comme valeur moyenne de l'excrétion d'azote métabolique fécal, chez le jeune Rat soumis à un régime à 4 p. Ioo de protéines d'œuf : I,I64 mg par gramme 
sec ingéré. Mais, les travaux ultérieurs de MITCHELL et BERT (I954) ayant mis en évidence l'existence d'une corrélation positive entre la teneur en protéines du régime et la concentration de la masse fécale en azote métabolique, MITchel lui-même (I962) retient plus récemment comme valeurs moyennes: I,3 à I,4 mg d'azote par gramme sec ingéré en régime protéiprive, I,5 à $\mathrm{I}, 9 \mathrm{mg}$ en régime à $4 \mathrm{p}$. Ioo de protéines d'œuf.

Tout en reconnaissant, chez le Rat, l'existence d'une corrélation positive entre 1'excrétion d'azote métabolique fécal et la matière sèche ingérée, MEYER (I956) a constaté que cette excrétion est en relation plus étroite avec la matière sèche fécale. Un fait analogue a été observé par HEUPKE (I933) chez 1'Homme, MUKERJEE et KeHAR (I948) chez le Bœuf, Blaxter et WoOd (I95I) chez le Veau, ZELTER et CharLET-LERT (I96I) chez le Porc.

$2^{\circ}$ En ce qui concerne l'excrétion d'azote endogène urinaire, il est largement reconnu qu'elle est en relation étroite - chez le Rat comme chez d'autres espèces animales - avec le poids corporel et avec le métabolisme basal, donc avec la puissance 0,70 ou 0,72 de ce poids (Terroine et Sorg MatTer, I927; Brody et al., I934; SMu's, I935; Mitcheis, I962) (1). Se basant sur cette relation, Brody et al. ont proposé la formule:

$$
\mathrm{N} \text { endogène urinaire }=\mathrm{I}_{4} 6 \mathrm{P} \quad 0,72
$$

Disposant de plus de 400 déterminations individuelles, réalisées chez de jeunes rats soumis à un régime protéiprive dans des conditions expérimentales standardisées, il nous a paru intéressant d'étudier à notre tour, l'influence de facteurs tels que le poids corporel, le poids des ingesta, ou celui des fèces, sur les pertes d'azote. Ceci, en vue :

- de comparer nos résultats à ceux qui ont été rapportés plus haut ;

- d'envisager simultanément - et non plus séparément - les relations entre l'excrétion d'azote métabolique fécal (out d'azote endogène urinaire) et plusieurs des facteurs susceptibles d'exercer une influence sur cette excrétion;

- de savoir dans quelle mesure les données établies peuvent être utilisées pratiquement dans 1'évaluation de 1'utilisation digestive ou de la valeur biologique des protéines.

Nous ne présenterons ici que les résultats obtenus chez de jeunes Rats soumis à un régime protéiprive. Une autre étude, réalisée avec un régime à $4 \mathrm{p}$. Ioo de protéines d'œuf, sera publiée ultérieurement.

\section{MÉTHODES EXPÉRIMENTALES}

Pour notre étude, nous avons utilisé les résultats de ro expériences réparties sur une période de 4 années. Ces expériences ont porté au total sur 4 I 7 rats mâles Wistar âgés de 5 à 6 semaines, provenant de l'élevage du Laboratoire. Ces animaux pesaient initialement de 70 à r 30 grammes (l'écart entre la plus forte et la plus faible valeur des poids étant de l'ordre de 30 grammes à l'intérieur de chacun des ro groupes expérimentaux).

() Brody (I945) désigne P 0,70 sous le vocable de "poids physiologique " ou de " taille métabolique '. 
Les expériences ont été standardisées sur les points suivants : rience $\left({ }^{(1)}\right.$;

- animaux de même souche, ayant reçu le même régime d'élevage avant la mise en expé-

- régime protéiprive standard, à base d'amidon (50 p. 100), de saccharose ( $32 \mathrm{p}$. roo), d'huile d'arachide ( 22 p. гоo), de mélange salin de HubBes et coll. (4 p. I00), d'agar-agar (2 p. roo), et de vitamines hydro et liposolubles $\left(^{2}\right)$. Ce régime contient de 38 à $42 \mathrm{mg}$ d'azote p. Ioo grammes de matière sèche; il est présenté sous forme de bouillie préparée avec I partie d'eau pour 2 parties de mélange sec;

- consommation de nourriture et d'eau distillée al libitum;

- conduite identique du bilan : après une période préliminaire de 2 jours, détermination des ingesta et récolte des fèces pendant 6 jours, en utilisant des dispositifs expérimentaux décrits par CAUSEReT (I954), placés dans une salle où la température atteint $22^{\circ} \pm \mathrm{I}, 5^{\circ}$;

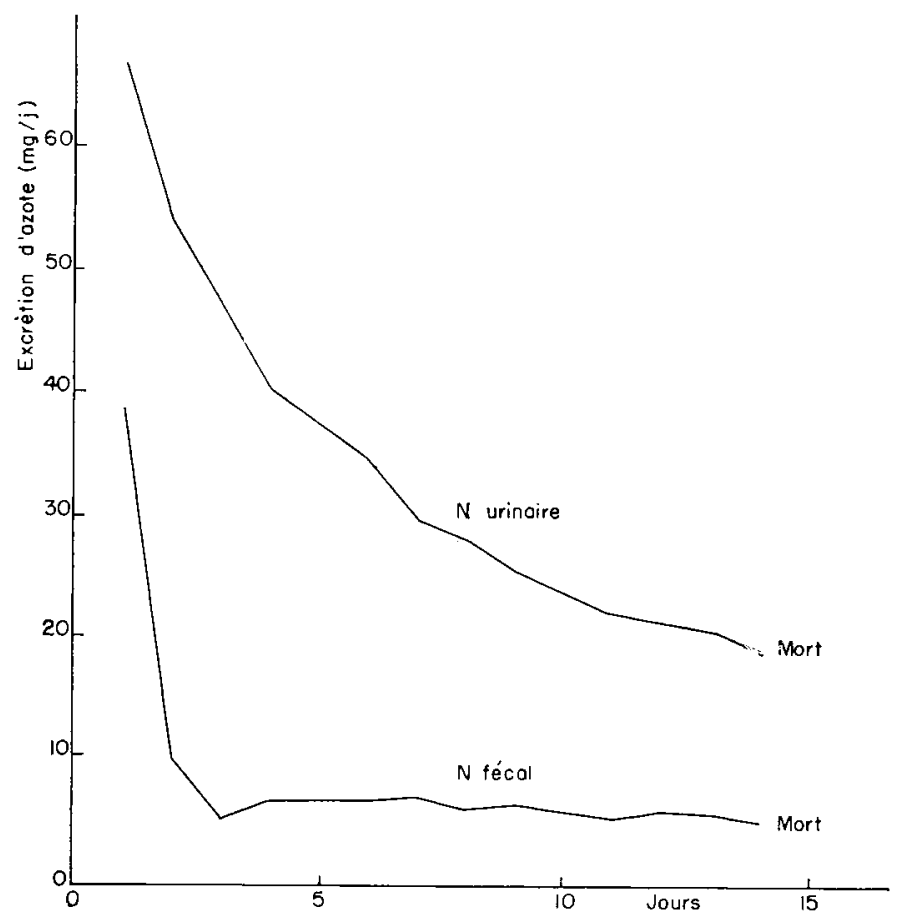

FIG. I. - Ezolution des excrétions fécale et urinaire d'azole au cours de l'inanition azotée (mojennes élablies sur 10 rats máles de 80 d 100 grammes)

Les quantités d'azote éliminées ont été évaluées par analyse d'une partie aliquote des fèces et des urines de chaque sujet (microkjeldahl).

La durée très courte de la période préliminaire a été imposée par la brièveté de la durée de survie des rats jeunes soumis au régime protéiprive : en général, la mort se produit dans un délai de I 2 à I5 jours, avec une importante perte de poids. On voit (fig. I) que les deux jours de période préliminaire suffisent à assurer la régularisation de l'excrétion fécale d'azote, mais non pas celle de l'excrétion urinaire, qui s'abaisse pendant toute la durée de la survie. Les valeurs de l'excrétion d'azote urinaire déterminées dans notre étude sont donc supérieures à la valeur ninimum atteinte dans les jours qui précèdent la mort. Est-ce cette dernière valeur qui correspond réellement à l'excrétion

(1) Biscuits " lixtralabo".

(2) Par $\mathrm{kg}$ de régime : thiamine, $2 \mathrm{mg}$; ribollavine $3 \mathrm{mg}$; pyrodoxine, $2,5 \mathrm{mg}$; acide nicotinique, $50 \mathrm{mg}$; pantothénate de calcium, Io $\mathrm{mg}$; vitanine $\mathrm{K}$ hydro-soluble, $\mathrm{I}$ my. L'huile d'arachide du régime est additionnée de 1 p. Ioo d'huile de foie de morue, titrant 2000 UI de vitamine A et 500 UI de vitimine D par ml, et de 0,015 p. 100 de tocophérol. 
endogène? Il est permis d'en douter, car les animaux perdent alors du poids très rapidement. On peut donc se ranger au point de vue de Mrtchell (I948) lorsqu'il écrit : "The lowest possible level of the urinary nitrogen can be obtained only after severe protein depletion and obviously bears no relation to the nitrogen requirement of maintenance under conditions of adequate nutrition."

\section{ÉTUDE STATISTIQUE DES RÉSULTATS}

Sur tous les animaux, nous avons déterminé expérimentalement:

l'excrétion d'azote métabolique fécal (mg par jour) ....... $y_{1}$

l'excrétion d'azote endogène urinaire (mg par jour) $\ldots \ldots$. $y_{2}$

le poids corporel initial (g) ..................... $x_{1}$

le poids de matière sèche ingérée (g par jour) .......... $x_{2}$

le poids de matière sèche fécale $\left(\mathrm{g}\right.$ par jour) ........... $x_{3}$

Le nombre des données expérimentales dépassant 2000 , il n'est pas possible de les reproduire ici ${ }^{(1)}$.

\section{A. Variations de l'azote métabolique fécal}

$\mathrm{I}^{0}$ Étude des régressions simples par groupe expérimental.

Pour chacun des dix groupes, les diagrammes de dispersion $\left({ }^{2}\right)$ obtenus en portant en ordonnée les valeurs de $y_{1}$, et en abscisse celles de $x_{1}$, de $x_{1}{ }^{0,70}$, de $x_{2}$ ou de $x_{3}$, suggèrent que ces variations sont linéaires. Nous avons donc calculé, à partir des données expérimentales, les équations des droites de régression correspondantes, ainsi que les valeurs du paramètre $F$ (SNÉDÉCOR) qui permettent de juger de la valeur des ajustements obtenus.

Ces données sont réunies dans le tableau I. Elles montrent que, pour presque tous les groupes, il existe des corrélations positives entre $y_{1}$ et chacune des variables indépendantes envisagées. La corrélation entre $y_{1}$ et $x_{3}$ est toujours hautement significative, tandis que la corrélation entre $y_{1}$ et $x_{1}\left(\right.$ or1 $\left.x_{1}{ }^{0,70}\right)$ est généralement la moins significative. Quant à la corrélation entre $y_{1}$ et $x_{2}$, sa signification est en général intermédiaire et varie beaucoup d'un groupe à un autre $\left.{ }^{3}\right)$.

Des diverses variables indépendantes envisagées, la matière sèche fécale $\left(x_{3}\right)$ apparaît donc comme la base d'estimation de l'azote métabolique fécal la plus satisfaisante. $2^{\circ}$ Existe-t-il des lois de régression communes à l'ensemble des groupes?

D'un groupe à l'autre, les paramètres des droites de régression de $y_{1}$ en $x_{1}$ (ou $x_{1}^{0,70}$ ), de $y_{1}$ en $x_{2}$, de $y_{1}$ en $x_{3}$ sont différents. Pour savoir s'il s'agit ou non de sim-

(i) Nous les ferons parvenir sous forme de tiré-ì-part, aux lecteurs qui désireraient en prendre connaissance.

(2) Non reproduits ici.

(3) Les variations de $y_{1}$ en fonction de l' "état de réplétion" du tube digestif $\left(\mathrm{R}=\frac{x_{2}}{x_{1}{ }^{0,70}}\right)$ ont aussi été étudiées ; comme $x_{2}$, R exprime le niveau d'ingestion de la matière sèche, mais certains nutritionnistes préfèrent ce mode d'expression, qui a l'avantage de faciliter les comparaisons entre sujets de poids différents. Toutefois, dans la présente étude, la corrélation entre $y_{1}$ et $R$ est, dans tous les groupes, moins significative que la corrélation entre $y_{1}$ et $x_{2}$.

Annales de Biologie animale. - 1965. 


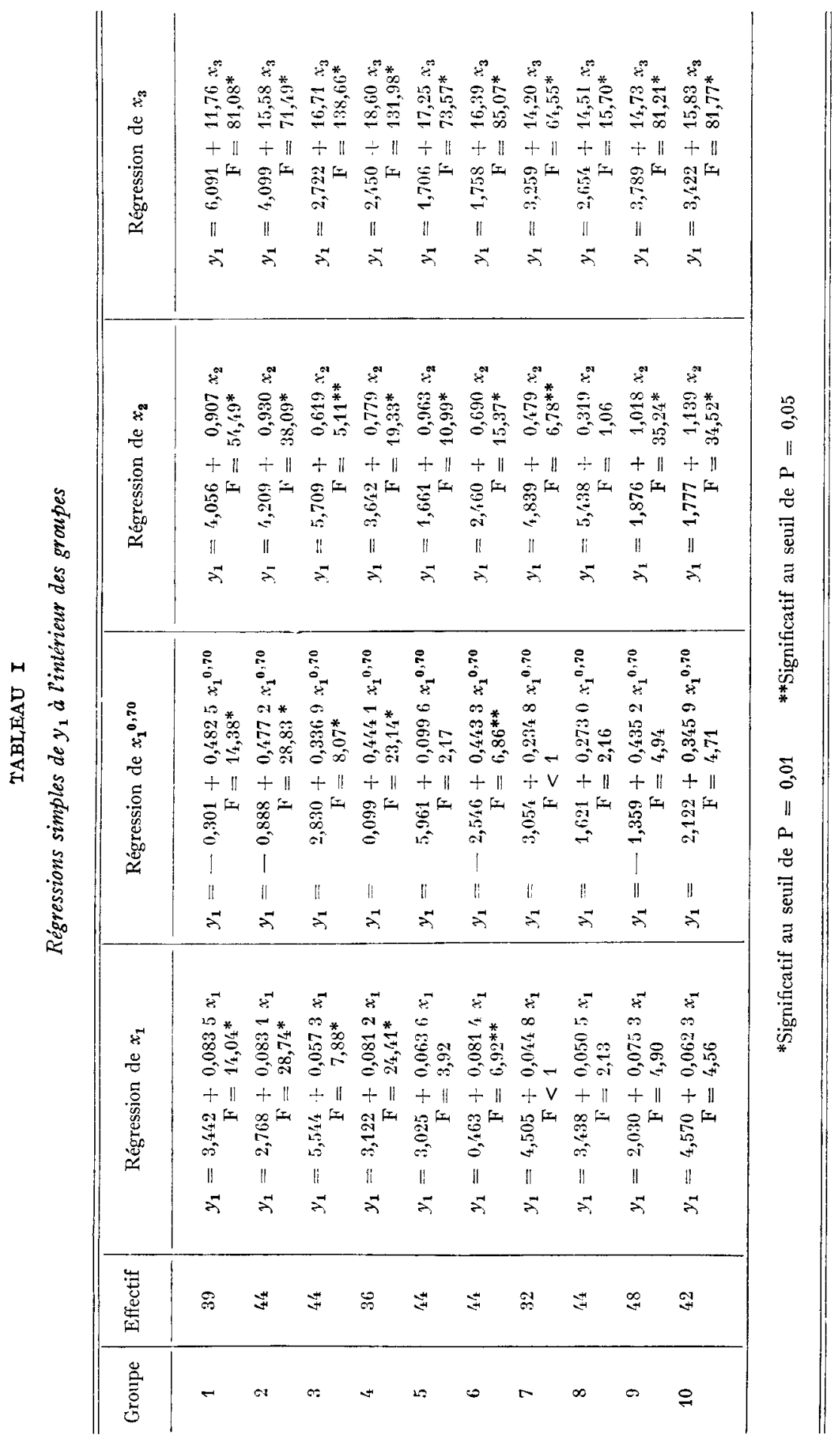


ples fluctuations dues au hasard de l'échantillonnage, nous avons vérifié l'homogénéité des ro valeurs de $b_{1}, b_{2}$ ou $b_{3}$ dans les équations:

$$
\begin{array}{ll}
y_{1}=a_{1}+b_{1} x_{1} & y_{1}=a_{1}^{\prime}+b^{\prime}{ }_{1} x_{1}{ }^{0,70} \\
y_{1}=a_{2}+b_{2} x_{2} & y_{1}=a_{3}+b_{3} x_{3}
\end{array}
$$

en appliquant le test $\mathrm{F}$ au rapport de la variance due aux différences entre les coefficients de régression à la variance de l'erreur. Quelle que soit la variable indépendante, ce test prouve qu'il n'y a pas d'hétérogénéité entre les $b$ des différents groupes.

En conséquence, il est possible de calculer la régression commune aux dix groupes. Cette estimation utilise l'ensemble des données de base, mais tient compte des écarts de ces données aux moyennes de chaque groupe, et non à la moyenne générale : on a donc considéré les sommes de carrés et de produits " résiduelles " fondées sur 407 degrés de liberté et non sur 4I6, la différence entre les deux valeurs correspondant aux ro moyennes de groupes.

Dans ces conditions, on obtient les équations générales :

$$
\begin{aligned}
& y_{1}= 3,065+0,07 \mathrm{I} I x_{1}, \\
&\text { avec } \mathrm{F}=89,7 \text { (significatif au seuil de } \mathrm{P}=0, \mathrm{OI}) \\
& y_{1}= 2,057+0,3 \mathrm{I} 97 x_{1}{ }^{\mathbf{0}, 70} \\
&\text { avec } \mathrm{F}=7 \mathrm{I}, 9 \text { (significatif au seuil de } \mathrm{P}=0, \mathrm{OI}) \\
& y_{1}= 3,4 \mathrm{I} 4+0,805 x_{2} \\
&\text { avec } \mathrm{F}=\mathrm{I} 58,3 \text { (significatif au seuil de } \mathrm{P}=0, \mathrm{OI}) \\
& y_{1}= 3,297+\mathrm{I} 5,27 x_{3} \\
&\text { avec } \mathrm{F}=689,2 \text { (significatif au seuil de } \mathrm{P}=\mathrm{O}, \mathrm{OI})
\end{aligned}
$$

Comme à l'intérieur de chaque groupe, c'est donc la corrélation entre l'excrétion d'azote métabolique fécal et le poids de matière sèche fécale qui est la plus forte.

$3^{\circ}$ Peut-on admettre la proportionnalité entre l'excrétion d'azote fécal métabolique et le poids du corps, le poids de matière sèche ingérée ou le poids de matière sèche fécale?

Dans les équations générales (I) à (4), les ordonnées à l'origine ont une valeur assez élevée par rapport aux valeurs expérimentales de $y_{1}$. Pour vérifier qu'elles sont significativement différentes de zéro, c'est-à-dire que les paramètres $b_{1}=0,07 \mathrm{I}$, $b_{1}^{\prime}=0,3 \operatorname{Ig} 7, b_{2}=0,805, b_{3}=I_{5}, 27$ ne peuvent être considérés comme des estimations de

$$
\beta_{1}=\frac{y_{1}}{x_{1}}, \quad \beta_{1}^{\prime}=\frac{y_{1}}{x_{1}^{0,70}}, \quad \beta_{2}=\frac{y_{1}}{x_{2}}, \quad \beta_{3}=\frac{y_{1}}{x_{3}},
$$

nous avons calculé les quatre paramètres :

$t_{1}=\frac{b_{1}-\beta_{1}}{\mathrm{~V} b_{1}+\mathrm{V} \beta_{1}} \quad t_{1}^{\prime}=\frac{b_{1}^{\prime}-\beta_{1}^{\prime}}{\mathrm{V} b_{1}^{\prime}+\mathrm{V} \beta_{1}^{\prime}} \quad t_{2}=\frac{b_{2}-\beta_{2}}{V b_{2}-V \beta_{2}} \quad t_{3}=\frac{b_{3}-\beta_{3}}{V b_{3}+V \beta_{3}}$ où $\mathrm{V} b_{1}, \mathrm{~V} b_{1}^{\prime}, \mathrm{V} b_{2}, \mathrm{~V} b_{3}, \mathrm{~V} \beta_{1}, \mathrm{~V} \beta_{1}^{\prime}, \mathrm{V} \beta_{2}, \mathrm{~V} \beta_{3}$ sont respectivement les variances de $b_{1}$, $b_{1}^{\prime}, b_{2}, b_{3}, \beta_{1}, \beta_{1}^{\prime}, \beta_{2}$ et $\beta_{3}\left(\right.$ avec $\mathrm{V} \beta_{1}=\mathrm{V} \beta_{2}=\mathrm{V} \beta_{3}=0$ ).

Tous ces paramètres sont significatifs au seuil de $\mathrm{P}=0,0$ I. Il n'y a donc pas de proportionnalité entre $y_{1}$ et chacune des variables indépendantes $x_{1}, x_{1}{ }^{0,70}, x_{2}, x_{3}$ : 
quelle que soit la base d'estimation adoptée pour $y_{1}$, l'excrétion d'azote métabolique fécal comprend toujours deux fractions, dont l'une seulement - la plus importante d'ailleurs - est proportionnelle à la variable indépendante envisagée.

$4^{\circ}$ Étude des régressions doubles et de la régression triple de l'excrétion d'azote métabolique fécal.

D'après ce qui précède, c'est le poids de matière sèche fécale qui fournit la meilleure base pour l'évaluation de l'azote métabolique fécal lorsqu'on n'envisage que les régressions simples de ce dernier. Mais il reste à savoir si l'on peut se contenter de cette base, ou s'il est préférable de tenir compte en même temps du poids corporel et du poids de matière sèche ingéré.

Nous avons donc étudié les diverses régressions doubles de $y_{1}$ en $x_{1}$ et $x_{2}$, en $x_{1}$

TABLEAU 2

Analyse progressive de la variance des régressions doubles de $y_{1}$ sur l'ensemble des groupes

\begin{tabular}{|c|c|c|c|c|c|c|c|}
\hline \multirow[b]{2}{*}{. } & \multirow{2}{*}{$\begin{array}{c}\text { Degrés de } \\
\text { liberté }\end{array}$} & \multicolumn{2}{|c|}{ Régression de $x_{3}$ et $x_{2}$} & \multicolumn{2}{|c|}{ Régression de $x_{3}$ et $x_{1}$} & \multicolumn{2}{|c|}{ Régression de $x_{2}$ et $x_{1}$} \\
\hline & & Variance & $\mathrm{F}$ & Variance & $\mathrm{F}$ & Variance & $F$ \\
\hline Régression de $x_{3} \ldots$ & 1 & 685,60 & 704,91 & 685,60 & $70 \div, 99$ & - & - \\
\hline Régression de $x_{2}\left({ }^{*}\right)$. & $i$ & 9,96 & 10,24 & - & $\ldots$ & 305,65 & 166,66 \\
\hline Régression de $x_{1}(*)$. & 1 & - & - & 10,00 & 10,28 & 11,07 & 22,39 \\
\hline Résidu ........... & $40 \%$ & 0,97 & - & 0,97 & - & 1,83 & - \\
\hline
\end{tabular}

\begin{tabular}{|c|c|c|c|c|c|c|c|}
\hline & \multirow{2}{*}{$\begin{array}{c}\text { Degrés de } \\
\text { liberté }\end{array}$} & \multicolumn{2}{|c|}{ Régression de $x_{2}$ et $x_{3}$} & \multicolumn{2}{|c|}{ Régression de $x_{1}$ et $x_{3}$} & \multicolumn{2}{|c|}{ Régression de $x_{1}$ et $x_{2}$} \\
\hline & & Variance & $\mathrm{F}$ & Variance & $\mathrm{F}$ & Variance & $\mathbf{F}$ \\
\hline Régression de $x_{1} \ldots$ & 1 & - & - & 197,17 & 202,75 & 197,17 & 107,51 \\
\hline Régression de $x_{2}\left({ }^{*}\right)$. & 1 & 305,65 & $31^{\prime}, 2^{\prime} \mathbf{t}$ & - & $\ldots$. & 149,55 & 81,54 \\
\hline Régression de $x_{3}\left(^{*}\right)$ & 1 & 390,91 & 401,92 & 498,43 & 512,52 & $\ldots$ & - \\
\hline Résidu $\ldots \ldots \ldots \ldots$ & $40^{\prime}$ & 0,97 & - & 0,92 & - & 1,83 & - \\
\hline
\end{tabular}

(*) Aprìs ajustement des variables déjà utilisées.

et $x_{3}$, en $x_{2}$ et $x_{3}$ et la régression triple de $y_{1}$ et $x_{1}, x_{2}$ en $x_{3}$. Dans cette étude, nous n'avons pas tenu compte de $x_{1}{ }^{0,70}$, les régressions simples ayant montré que la précision des estimations obtenues avec cette variable n'est pas plus élevée qu'avec $x_{1}$.

En ce qui concerne les régressions doubles, l'analyse progressive des variances montre que, lorsqu'on a considéré l'une quelconque des variables indépendantes $x_{1}, x_{2}$ ou $x_{3}$, il y a toujours intérêt à tenir compte également de l'une ou de l'autre des deux variables restantes (tabl. 2). Ce sont les régressions en $x_{3}$ et $x_{1}$, ou en $x_{3}$ et 
$x_{2}$ qui expriment les variations de $y_{1}$ de la manière la plus valable. En effet, le paramètre $\mathrm{F}$ atteint respectivement:

$$
\begin{aligned}
& \mathrm{F}=94,5 \text { pour la régression de } y_{1} \text { en } x_{2} \text { et } x_{1} \\
& \mathrm{~F}=357,6 \text { pour la régression de } y_{1} \text { en } x_{3} \text { et } x_{1} \\
& \mathrm{~F}=357,6 \text { pour la régression de } y_{1} \text { en } x_{3} \text { et } x_{2}
\end{aligned}
$$

Cependant, l'analyse de variance de la régression iriple montre que, lorsqu'on a considéré deux quelconques des trois variables indépendantes, il y a toujours inté-

\begin{tabular}{|c|c|c|c|c|c|c|c|}
\hline & \multirow{2}{*}{$\begin{array}{c}\text { Degrés } \\
\text { de } \\
\text { liberté }\end{array}$} & \multicolumn{2}{|c|}{$\begin{array}{l}\text { Régression } \\
\text { de } x_{3}, x_{1} \text { et } x_{2}\end{array}$} & & \multirow{2}{*}{$\begin{array}{c}\text { Degrés } \\
\text { de } \\
\text { liberté }\end{array}$} & \multicolumn{2}{|c|}{$\begin{array}{c}\text { Régression } \\
\text { de } x_{3}, x_{2} \text { et } x_{1}\end{array}$} \\
\hline & & Variance & $\mathrm{F}$ & & & Variance & $\mathbf{F}$ \\
\hline 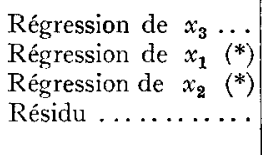 & $\begin{array}{c}1 \\
1 \\
1 \\
404\end{array}$ & $\begin{array}{r}685,60 \\
10,00 \\
15,13 \\
0,94\end{array}$ & $\begin{array}{r}731,31 \\
10,67 \\
16,14 \\
-\end{array}$ & 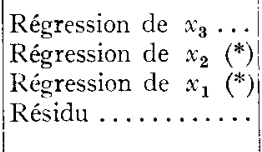 & $\begin{array}{c}1 \\
1 \\
1 \\
404\end{array}$ & $\begin{array}{r}685,60 \\
9,96 \\
15,17 \\
0,94\end{array}$ & $\begin{array}{r}731,31 \\
10,62 \\
16,18 \\
\end{array}$ \\
\hline
\end{tabular}

TABLEAU 3

Analyse progressive de la variance de la régression triple de $y_{1}$ sur l'ensemble des groupes

(*) Après ajustement des variables déjà utilisées.

rêt à tenir compte également de la troisième (tab1. 3). Comme c'est avec $x_{3}$ que la relation de $y_{1}$ est la plus étroite, nous reproduisons simplement les éléments de l'analyse correspondant aux régressions de $x_{1}$ et de $x_{2}$ après ajustement de $x_{3}$.

L'équation de régression triple est:

$$
y_{1}=\mathrm{I}, 722+0,04 \mathrm{I} 4 x_{1}-0,4 \mathrm{I} 5 x_{2}+\mathrm{I} 7,576 x_{3}
$$

$5^{\circ}$ Équations de régression générales et familles d'équations de groupes.

Parmi les équations générales étudiées, les équations (4) et (5) sont celles qui permettent d'estimer l'excrétion d'azote métabolique fécal avec le maximum de précision.

Mais, puisqu'il existe des lois de régression communes à tous les groupes, ce sont en réalité des familles de droites ne différant les unes des autres que par la valeur de leur ordonnée à l'origine qui représentent les phénomènes observés avec le plus d'exactitude (à titre d'exemple, voir la fig. 2).

Pour le calcul de l'excrétion d'azote métabolique fécal, il apparaît donc préférable de substituer aux équations générales (4) ou (5) une "famille "d'équations de la forme:

$$
\begin{aligned}
& y_{1}=a+\mathrm{I} 5,27 x_{3} \\
& y_{1}=a+0,04 \mathrm{I} 4 x_{1}-0,4 \mathrm{I} 5 x_{2}+\mathrm{I} 7,57^{6} x_{3}
\end{aligned}
$$


Les valeurs du paramètre $a$ sont calculées pour chaque groupe en attribuant à $y_{1}, x_{1}, x_{2}$ et $x_{3}$ leurs valeurs moyennes à l'intérieur du groupe :

Valeur du paramètre $a$

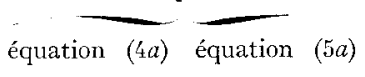

$\begin{array}{lll}\text { Groupe } 1 \ldots & - & - \\ \text { Groupe } 2 \ldots & 4,36 & 2,70 \\ \text { Groupe } 3 \ldots & 1,25 & 2,11 \\ \text { Groupe } 4 \ldots & 3,48 & 0,82 \\ \text { Groupe } 5 \ldots & 3,92 & 2,78 \\ & 2,14 & 1,07\end{array}$

Valeur du paramètre $a$

équation (4a) équation (5a)

$\begin{array}{cccc}\text { Groupe } & 6 \ldots & 2,13 & 0,77 \\ \text { Groupe } & 7 \ldots & 2,86 & 1,52 \\ \text { Groupe } & 8 \ldots & 2,39 & 1,18 \\ \text { Groupe } & 9 \ldots & 3,57 & 1,66 \\ \text { Groupe } 10 \ldots & 3,65 & 2,14\end{array}$

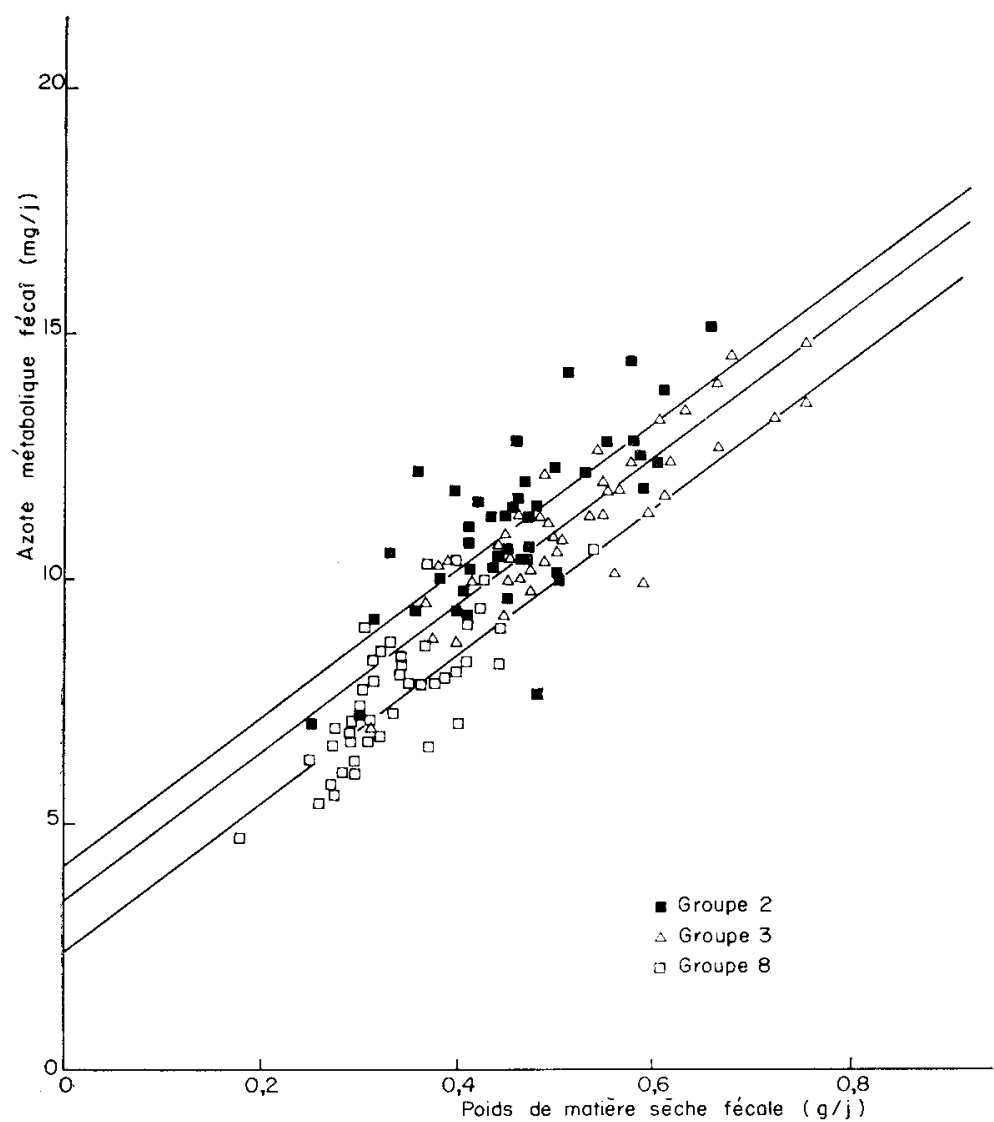

FIc.2. - Droites de régression $\mathrm{y}_{1}=\mathrm{a}+b x_{3}$ pour trois groupes expérimentaux (à titre d'exemple) (de haut en bas, les trois droites de régression concernent respectivement les groupes 23 et 8).

\section{B. Variations de l'azote endogène urinaire}

I Étude des régressions simples par yroupe expérimental.

Pour chacun des dix groupes, les diagrammes de dispersion $\left(^{1}\right)$ obtenus en portant en ordonnée les valeurs de $y_{2}$ et en abscisse celles de $x_{1}$, de $x_{1}{ }^{0,70}$ et de $x_{2}$, suggèrent que ces variations sont linéaires.

(1) Non reproduits ici. 
AZOTE MÉTABOLIQUE FÉCAL ET ENDOGÈNE URINAIRE,

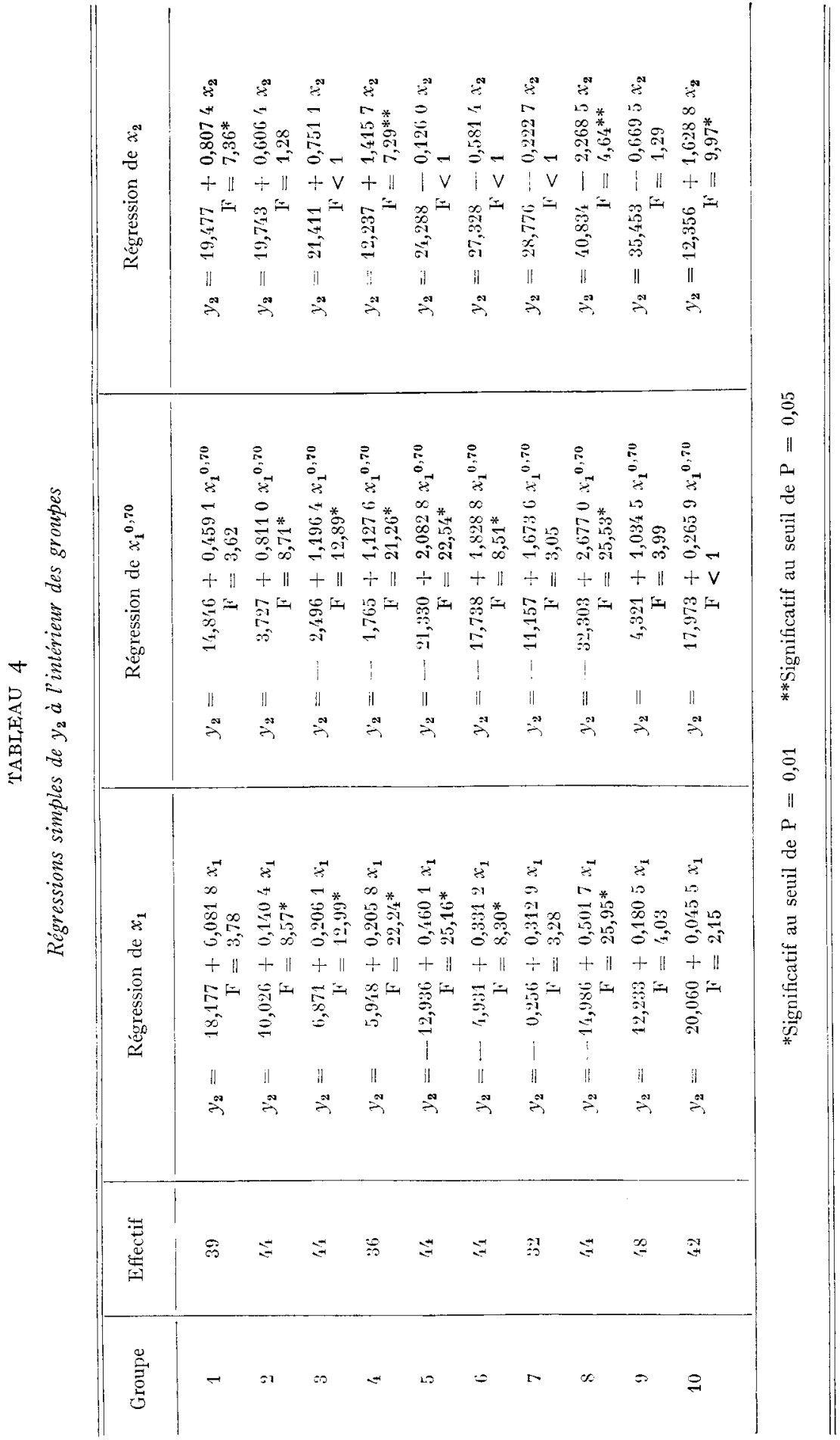


Les données du tableau 4, qui sont présentées de la même manière que celles du tableau I, montrent qu'il existe effectivement, dans la plupart des groupes, des corrélations positives significatives entre $y_{2}$ et $x_{1}$ ou $x_{1}{ }^{0,70}$.

Il existe également des corrélations plus faibles, et plus rarement significatives, entre $y_{2}$ et $x_{2}$.

\section{$2^{\circ}$ Existe-t-il des lois de régression communes à l'ensemble des groupes?}

L'application du test $\mathrm{F}$, dans les mêmes conditions que celles concernant la variable $y_{1}$, aux Io valeurs de $b_{1}$ ou $b_{2}$ dans les équations $y_{2}=a_{1}+b_{1} x_{1}$, $y_{2}=a_{1}^{\prime}+b_{1}^{\prime} x_{1}{ }^{0,70} \quad y_{2}=a_{2}+b_{2} x_{2}$ prouve qu'il n'y a pas d'hétérogénéité entre les $b$ des différents groupes.

Comme en ce qui concerne l'azote fécal métabolique, nous avons donc calculé les régressions communes aux Io groupes dans les mêmes conditions :

$$
\begin{aligned}
y_{2}= & 6,728+0,2077 x_{1} \\
& \text { avec } \mathrm{F}=9 \mathrm{I}, 7 \text { (significatif au seuil de } \mathrm{P}=\mathrm{O}, \mathrm{OI}) \\
y_{2}= & 6,289+0,0279 x_{1}^{0,70} \\
& \text { avec } \mathrm{F}=55,5 \text { (significatif au seuil de } \mathrm{P}=\mathrm{O}, \mathrm{OI}) \\
y_{2}= & 23,522+0,3002 x_{2} \\
& \text { avec } \mathrm{F}=\mathrm{I}, 90 \text { (non significatif) }
\end{aligned}
$$

On voit beaucoup plus nettement qu'à l'intérieur de chaque groupe, que le poids corporel des animaux (ou leur " taille métabolique " constitue la seule base d'estimation valable pour l'excrétion d'azote endogène urinaire. Dans la suite de cette étude, c'est au poids que nous accorderons la préférence, puisque la régression de $y_{2}$ en $x_{1}{ }^{0,70}$ n'est pas plus significative que la régression de $y_{2}$ en $x_{1}$.

\section{TABLEAU 5}

Analyse progressive de la variance de la régression double de $y_{2}$ sur l'ensemble des groupes

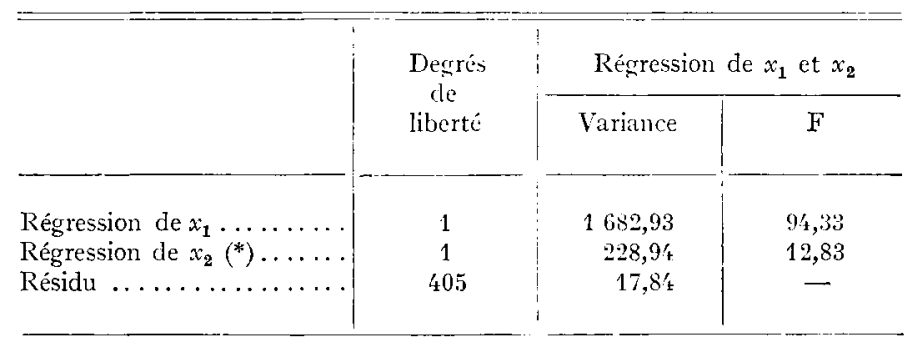

(*) Après ajustement de la variable déjà utilisée.

$3^{\circ}$ Peut-on admettre la proportionnalité entre l'excrétion d'azote urinaire endogène et le poids du corps?

L'étude, conduite dans les mêmes conditions que pour les régressions simples de $y_{1}$, permet de répondre négativement à cette question. 
L'excrétion d'azote endogène urinaire comprend donc deux fractions, dont l'une seulement - la plus importante - est proportionnelle au poids du corps.

$4^{0}$ Étude de la régression double de l'excrétion d'azote endogène urinaire.

Bien que la corrélation entre $y_{2}$ et $x_{2}$ soit faible, il a paru intéressant d'étudier la régression double de $y_{2}$ en $x_{1}$ et $x_{2}$.

L'analyse progressive des variances montre que, lorsqu'on a considéré la variable indépendante $x_{1}$, il y a intérêt à tenir compte également de $x_{2}$ (tabl. 5). La valeur de $F$ est de 53,6.

L'équation de régression double est:

$$
y_{2}=8,966+0,2498 x_{1}-0,7956 x_{2}
$$

$5^{\circ}$ Équations de régression générales et familles d'équations de groupes.

Comme pour le calcul de l'excrétion d'azote métabolique fécal, il y a intérêt à substituer aux équations générales (6) ou (9), une "famille " d'équations de la forme :

$$
\begin{aligned}
& y_{2}=a+0,2077 x_{1} \\
& y_{2}=a+0,2498 x_{1}-0,7956 x_{2}
\end{aligned}
$$

Les valeurs du paramètre $a$ sont calculées pour chaque groupe en attribuant à $y_{2}$, $x_{1}$ et $x_{2}$ leurs valeurs moyennes à l'intérieur du groupe :

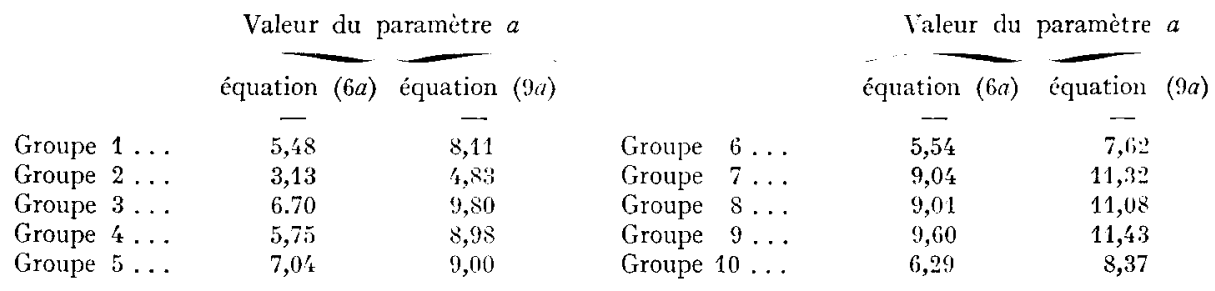

\section{DISCUSSION}

\section{A. Azote métabolique fécal}

a) D'après ce qui précède, le calcul de l'excrétion d'azote métabolique fécal sur la seule base d'une valeur standard par gramme de matière sèche ingérée ou de matière sèche fécale paraît difficilement applicable chez le jeune Rat. Si, en négligeant le fait que l'ordonnée à l'origine des équations de régression simple (2) et (3) est significativement différente de zéro, on substitue à ces équations celles de droites passant par l'origine et par le point de coordonnées $\left(\bar{x}_{2}, \bar{y}_{1}\right)$ ou $\left(\bar{x}_{3}, \bar{y}_{1}\right)$, on introduit dans le calcul de l'excrétion d'azote fécal une erreur relative supplémentaire pouvant aller jusqu'à 20 p. roo chez les animaux dont la consommation de nourriture atteint les valeurs les plus faibles ou les plus élevées (1). Il est donc préférable de tenir compte du facteur constant des deux équations de régression.

(1) Signalons cependant que, chez nos animaux, la perte moyenne d'azote métabolique fécal par gramme de matière sèche ingérée atteint $\mathrm{I}, 25 \mathrm{mg}$ : exprimée sous cette forme, elle est donc voisine de celle que MrTECIIELL (I948) a obtenue chez le jeune Rat également soumis ì un régime protéiprive ( $1,3-\mathrm{I}, \mathrm{f} \mathrm{mg}$ ). 
b) L'existence d'une légère influence propre du poids corporel et du poids des ingesta sur la perte d'azote métabolique fécal, influence qui reste indépendante de celle que ces mêmes facteurs exercent par l'intermédiaire du poids des excreta, constitue un fait nouveau.

Ce fait ne peut être rapproché des résultats obtenus par d'autres expérimentateurs ayant travaillé sur le Rat, puisque aucun d'eux n'a étudié les variations doubles ou triples de l'excrétion d'azote métabolique fécal en fonction des facteurs envisagés. Rappelons cependant que, d'après les recherches de ZELTER et CHARLET-LÉRY (I96I) sur le Porc, le poids corporel n'aurait pas d'effet sur la concentration de la masse fécale en azote métabolique, mais seulement sur la valeur de cette masse : ce qui permet de conclure que l'estimation de l'excrétion d'azote métabolique fécal en fonction de la matière sèche fécale évite l'interférence du facteur poids, qui intervient lorsque l'estimation s'effectue par rapport à la matière sèche ingérée.

Il y a donc là une divergence de résultats qui peut s'expliquer soit par le nombre moins élevé d'animaux utilisé par ZEITER et CHARIET-LÉRY, soit par les différences entre les conditions des deux expériences (espèces animales différentes, importance de la chute de poids chez le Rat soumis à un réginı protéiprive alors que le Porc maintient son poids pendant plusieurs semaines, dispersion plus faible des valeurs individuelles du poids corporel initial dans notre travail, etc.).

\section{TABIEAU 6}

Ecarts entre valeurs calculées et valeurs expérimentales de l'excrétion d'asote mítabolique fécal (N.M.F.)

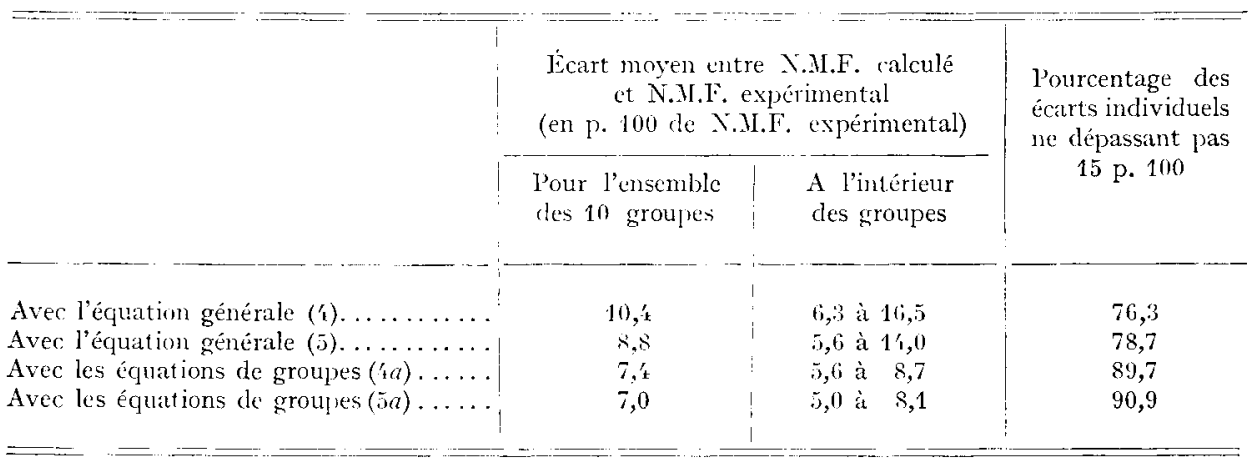

c) L'interprétation biologique de l'équation (5) pose un problème : il peut paraître surprenant que, lorsqu'on a tenu compte de l'influence du poids corporel et du poids des fèces, la part de l'influence du poids des ingesta qui reste indépendante du poids des fèces soit négative.

On peut cependant trouver une explication à ce phénomène si l'on se souvient:

- que les jeunes rats soumis au régime protéiprive consomment une quantité de nourriture irférieure de 20 à $25 \mathrm{p}$. Ioo à celle qu'ingèrent des sujets de même â ge et de même poids soumis à un régime complet;

- que cette sous-consonmation s'accompagne d'une perte de poids progressive, de l'ordre de 2 grammes par jour. 
Conme les réserves grasses du Rat jeune sont peu importantes, il est probable que l'organisme doit faire appel à ses protéines tissulaires pour couvrir une part de son besoin énergétique : en sorte qu'à poids corporel égal et à poids de fèces égal, les sujets dont la consommation de nourriture est la plus élevée - donc s'approche le plus de la consommation normale - épargnent mieux leurs protéines corporelles et excrètent moins d'azote.

d) Pour exprimer les résultats de notre étude de manière plus concrète, nous avons calculé les $4 \mathrm{I} 7$ valeuts individuelles de l'excrétion d'azote métabolique fécal au moyen des équations (4), (5), (4a), (5a) et nous avons comparé ces estimations aux valeurs expérimentales correspondantes. Ne pouvant reproduire ici toutes ces comparaisons, nous nous bornerons à en tirer quelques données (tab1. 6).

On voit que la substitution des équations de groupe $(4 a)$ ou $(5 a)$ aux équations générales (4) ou (5) accroît fortement la précision des estimations. Ce fait est particulièrement net à l'intérieur de certains groupes.

\section{B. Azote endogène urinaire}

a) En ce qui concerne l'évaluation de l'excrétion d'azote endogène urinaire, nos constatations confirment partiellement celles des auteurs qui nous ont précédés : le poids corporel constitue une base d'estimation valable. Mais il n'y a aucun intérêt à rapporter l'excrétion à la puissance 0,70 du poids corporel : les calculs sont aussi précis en la rapportant au poids lui-même $\left(^{1}\right)$.

\section{TABLEAU 7}

\section{Ĺcarls entre valeurs calculées et valeurs expérimentales de l'excrétion d'azole endogène urinaire (N.E. L.)}

Avec l'équation générale (i) $\ldots \ldots \ldots \ldots$ 14,

Avec l'équation générale $(10) \ldots \ldots \ldots \ldots . . \ldots$

Avec les équations de groupes $(6 a) \ldots \ldots$. 13,0

Avec les équations de groujes (10a) ... 12,9

Pourcentage des ecarts individuels ne dépassant pas $15 \mathrm{p} .100$

P'our l'ensemble des 10 groupes
60,0

$1 ; 1,1$

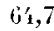

65,9

En outre, il est préférable de ne pas négliger l'ordonnée à l'origine de l'équation de régression (6). Si on substitue à cette dernière l'équation d'une droite passant par l'origine et par le point de coordonnées $\left(\bar{y}_{2}, \bar{x}_{1}\right)$, on introduit dans le calcul de l'excré-

(1) Il n'en serait peut-ĉtre pas de même si le poids corporel initial des animaux variait entre des limites plus larges que dans cette étude. 
tion d'azote endogène urinaire une erreur relative supplémentaire pouvant aller jusqu'à Io p. Ioo chez les animaux dont la consommation de nourriture atteint les valeurs les plus faibles ou les plus élevées.

b) Pour exprimer les résultats de notre étude de manière plus concrète, nous avons calculé les 4I7 valeurs individuelles de l'excrétion d'azote endogène urinaire au moyen des équations (6), (9), (6 a), (9a), et nous avons comparé ces estimations aux valeurs expérimentales correspondantes. Comme dans le cas de l'azote métabolique fécal, nous arons tiré de ces comparaisons, quelques données résumées dans le tableau 7 .

Bien que la substitution des équations de groupes $(6 a)$ ou $(9 a)$ aux équations générales correspondantes accroisse légèrement la précision des résultats obtenus, on voit que, dans les meilleures conditions, cette précision reste nettement inférieure à celle du calcul de l'azote métabolique fécal : à l'intérieur de certains groupes, la divergence entre valeurs calculées et valeurs observées est considérable.

c) Il est regrettable que la consommation d'eau et le volume urinaire n'aient pas été déterminés. Il eût été intéressant de chercher si l'excrétion d'azote urinaire ne dépend pas de ces deux facteurs, comme l'azote métabolique fécal dépend de la quantité de matière sèche ingérée et du poids sec des fèces. Nous reprendrons l'étude de ce problème sur de nouveaux groupes d'animaux.

\section{Titilisation des données de l'expérience pour l'évaluation de l'utilisation digestive et de la valeur biologique des protéines}

Dans l'évaluation du coefficient d'utilisation digestive réel et de la valeur biologique des protéines, est-il possible de remplacer la détermination expérimentale individuelle de l'excrétion d'azote métabolique fécal et d'azote endogène urinaire par des calculs basés sur les données contenues dans ce travail?

$A$ priori, on peut répondre négativement à cette question en ce qui concerne l'évaluation de l'excrétion d'azote endogène urinaire. En revanche, il paraît possible de calculer l'excrétion d'azote métabolique fécal à partir des équations (4a) ou (5 $a$ ), dans lesquelles on a donné au coefficient $a$ sa valeur particulière pour le groupe d'animaux considéré. Le calcul de cette valeur implique seulement:

- la détermination expérimentale du poids moyen des fèces $\left(\bar{x}_{3}\right)$, du poids moyen des ingesta $\left(\bar{x}_{2}\right)$ et du poids corporel initial moyen $\left(\bar{x}_{1}\right)$ à l'intérieur du groupe ;

- un dosage d'azote dans un échantillon moyen de fèces obtenu en mélangeant des parties aliquotes des fèces de tous les animaux du groupe, en vue d'en déduire l'excrétion moyenne d'azote métabolique fécal $\left(\bar{y}_{1}\right)$.

Nous avons donc comparé, pour nos 4I7 animaux, les coefficients d'utilisation digestive réels (C.U.D.) et les valeurs biologiques (V.B.) calculés sur des bases entièrement expérimentales, aux résultats obtenus en retenant pour le calcul les valeurs de l'excrétion d'azote métabolique fécal estimées à partir de l'équation $(5 a)$. 
Comme le prouve le tableau ci-dessous, la concordance entre les det1x séries de valeurs est satisfaisante:

$\begin{array}{cr}\text { Cas du } & \text { Cas de la } \\ \text { C.C.D. réel } & \text { V.B. } \\ - & - \\ & \\ 0,5 & 0,2 \\ 69,1 & 95,4 \\ 90, t & 98,7 \\ 97, t & 99,2 \\ 99,3 & 99,5 \\ 100,0 & 100,0\end{array}$

Nos études ultérieures montreront si le calcul de l'excrétion d'azote endogène urinaire en fonction de la consommation d'eau et du volume d'urine émis permet d'évaluer la V.B. des protéines sans déterminer expérimentalement cette excrétion.

Reçu pour publication en décembre r964.

\title{
SUMMARY
}

\begin{abstract}
STATISTICAL STUDY OF VARIATIONS IN EXCRETION OF METABOLIC NITROGEN
IN FACES AND ENDOGENOUS NITROGEN IN URINE IN RELATION TO BODY-WEIGHT, FEED INTAKE AND WEIGHT OF FACES IN THE YOUNG RAT.
\end{abstract}

\section{ON A PROTEIN-FREE DIET}

The influence of bodyweight, weight of dry-matter eaten and weight of dry matter of fæces on excretion of nitrogen was studied statistically in ro groups of young male rats weighing 70 to $13 \circ \mathrm{g}$ at the start, and given a protein-free diet. The groups, a total of $4 \mathrm{I} 7$ subjects, were put on test at different times spread over a period of four years.

a) In each group there was a positive correlation between metabolic nitrogen excreted in foces and each of the three independent variables listed above : however, the correlation between metabolic nitrogen in fæces and the weight of fæces was always the most highly significant. Whatever independant variable was used there was a form of regression common to all the groups.

Further, when the influence of any one of the three independent variables has been considered, it is of value to take the other two into account also, estimating the excretion of metabolic nitrogen in fæces on the basis of a triple regression equation.

Finally, whether the excretion is estimated from only one or from all three independent variables, the precision of the calculation is increased by using separate equations specific for each experimental group instead of that derived for all the animals combined : the equations differ from one another only in the value of the constant.

b) In some groups there was a positive correlation between endogenous nitrogen excreted in urine and bodyweight or weight of dry-matter eaten; the correlation with bodyweight was the more highly significant.

For the two independent variables there was, on the other hand, a form of regression common to all groups.

Excretion of endogenous nitrogen in urine was estimated most accurately from a double regression equation embodying the influence of both bodyweight and the weight of dry-matter eaten.

Finally, it is advantageous to use equations specific to each experimental group rather than that for all the animals together; the equations differ only in the value of the constant.

However, it was not possible to derive a function from which excretion in urine could be estimated as accurately as excretion in fæces. It might be asked whether more satisfactory bases for the estimation would be the intake of water and the volume of urine : that possibility is under consideration in experiments in progress. 
c) It would seem possible that information relating to the excretion of metabolic nitrogen in fæces could be used in estimation of true digestibility and biological value of proteins. On the other hand use of the data used for calculation of excretion of endogenous nitrogen in urine could lead to considerable error.

\section{RÉFÉRENCES BIBLIOGRAPHIQUES}

Blaxter K. L., Wood W. A., 1951. The nutrition of the young Ayrshire calf. I. The endogenous nitrogen and basal energy metabolism of the calf. Brit. J. Nutr., 5, i .

BoAs Fixsex M. A., Jackson H. M., I932. The biological values of proteins. IV. The biological values of the proteins of wheat, maize and milk. Biochem. J., 26, 1919-1923.

Brody S. R., Procter C., Ashworth U. S. Growth and development with special reference to domestic animals. XXXIV. Basal metabolism, endogenous nitrogen, creatinine and neutral sulfur excretions as functions of body weight. Missouri Agr. Expt. Sta. Research Bull., n ${ }^{0} 220$.

Brody S. R., 1945. Bioenergetics and Growth. Reinhold Publ. Co, New York.

CAuseret J., 1954. Recherches sur l'utilisation physiologique du calcium au cours de la croissance chez le Rat. Ann. Zootech., 3, 271-336.

Chick H., Hutchinson J. C. D., Jackson H. M., I935. The biological value of proteins. VI. Further investigation of the balance sheet method. Biochem. J., 29, I702-I7II.

Heupke W., I933. Über die Abhangigkeit der Menge des Kotstickstoffs von der Grösse des Stuhlrolumens. Arch. Hyg. Bekt., 111, I88.

MARTIN C., Robinson J., I 922 . Minimum N expenditure of man and the biological value of various proteins for human nutrition. Biochem. J., 18, 407 .

MEYER J. H., 1956. Influence of dietary fiber on metabolic and endogenous nitrogen excretion. $J$. Nutr., 58, $407-413$.

Mitchell H. H., I923-1924. A method of determining the biological value of a protein. J. Biol. Chem., 58, $873-903$.

Mitchell H. H., 1926. The determination of the requirements of animals and of the protein values of farm feeds and rations. Bull. Nat. Research Council $\mathrm{n}^{\circ} 55$.

Mrtchell H. H., 1948. The biological utilisation of proteins and protein requirements. In : Sahyun M. (Ed.). Proteins and amino acids in nutrition. Reinhold Publ. Co, New York, pp 46-8r.

Mitchell H. H., ig62. Comparative nutrition of man and domestic animals. Academic Press, New York. Pp 129-191.

Mitchell H. H., BerT M. H., I954. The determination of metabolic fecal nitrogen. J. Nutr., 52, 483-497,

Mukherjee R., Kemar N. D., 1948. Studies on protein metabolism. III. An improved technique for estimating the metabolic fecal nitrogen of cattle. Indian J.Vet. Sci., 19, 99-1 I 2.

SCHNEIDER B. H., 1934. The relationrhip of the metabolic nitrogen of the faeces to body weight and to food intake for rats. Biochem. J., 28, 360-364.

SmuTs D. B., 1935. The relation between the basal metabolism and the endogenous nitrogen metabolism with particular reference to the estimation of the maintenance requirement of protein. J. Nutr., 9, 403-433,

TERroine E. F., Sorg MatTer H., i927. Loi quantitative de la dépense azotée minima des homéothermes. Arch. Int. Physiol., 30, II 5-125.

Zelter S. Z., CharLet-LÉRY G., ig6r. Efficacité de quelques protides alimentaires chez le Porc. I. Excrétion d'azote métabolique fécal et endogène urinaire : influence du niveau d'ingestion de matière sèche, du poids corporel, de l'âge et de l'indigestible. Ann. Biol. anim. Bioch. Biophys., 1, 29-46. 